\title{
A commentary on Chen and Campbell (2017): Is there a clear case for addition fact recall?
}

\author{
Arthur J. Baroody ${ }^{1,2}$ \\ Published online: 5 March 2018 \\ (C) Psychonomic Society, Inc. 2018
}

\begin{abstract}
In their review of Uittenhove, Thevenot and Barrouillet (Cognition, 146, 289-303, 2016), Chen and Campbell (Psychonomic Bulletin \& Review, 2017 https://doi.org/10.3758/s13423-017-1328-2) concluded that existing evidence supports the conventional wisdom that basic non-zero addition combinations are stored and retrieved as discrete facts and that compacted reconstructive strategies play no role in expert mental addition. One aim of the present commentary is to detail why their evidence supporting these conclusions is not unequivocal. A second aim is to delineate key issues that still need to be addressed to build an accurate model of how basic sums are represented and retrieved.
\end{abstract}

Keywords Mental arithmetic $\cdot$ Recall $\cdot$ Reconstructive processes $\cdot$ Reasoning strategies

\section{Introduction}

How experts mentally represent and process basic addition combinations such as $1+7,3+3$, and $9+5$ has been debated for over three decades (e.g., Ashcraft, 1982; Baroody, 1983). The conventional wisdom in cognitive psychology has long been that fact recall (a reproductive process) replaces counting and reasoning strategies (reconstructive processes), because the former is more efficient than the latter (National Mathematics Advisory Panel, 2008). That, is, "true" mental-addition experts store all basic combinations not involving zero as a specific association between the addends and their sum and directly recall such facts. Recently, proponents of a compacted (automatic and non-conscious) counting model have revived the debate by suggesting that fast reconstructive processes play a role in the retrieval of small sums (Barrouillet \& Thevenot 2013). "Compacted" Procedures for Adults' Simple Addition" (Chen \& Campbell, 2017) is a timely and valuable review of this challenge to the current conventional wisdom. The authors' critique of Uittenhove, Thevenot and Barrouillet (2016) and related research supporting a compacted counting model makes many good points that proponents of the model need to address.

Arthur J. Baroody

baroody@illinois.edu

University of Illinois at Urbana-Champaign, Champaign, IL, USA

2 University of Denver, Denver, CO, USA
The aims of the present commentary, though, are not to evaluate the merits of the compacted counting model or Chen and Campbell's (2017) critique of its supporting data. One aim is to question Chen and Campbell's conclusion that the existing evidence clearly indicates that expert mental addition with non-zero items exclusively involves a reproductive process. A second aim of the commentary is to outline a number of key assumptions or unresolved issues that need to be empirically addressed to achieve an accurate picture of expert mental addition.

\section{Questions about Chen and Campbell's conclusion of an exclusive reproductive process}

The evidence supporting the current conventional wisdom that mental-addition experts exclusively rely on a reproductive process is, in fact, far from unequivocal. Moreover, Chen and Campbell's (2017) empirical case against any form of compacted addition procedures (reconstructive strategies) is also not clear-cut.

\section{Why the evidence supporting the current conventional wisdom is not unequivocal}

In constructing a model of expert retrieval, it is essential to distinguish among data generated by relatively slow, conscious, procedure-based processes (slow reconstructive 
strategies), relatively fast, non-conscious procedure-based processes (automated or compacted reconstructive processes), and a relatively fast and direct fact-recall process (reproductive process). ${ }^{1}$ Uittenhove et al. (2016) took steps to eliminate data derived from slow reconstructive strategy for very small problems. However, Chen and Campbell (2017) reasonably concluded on p. 6 that "there can be little doubt that [their] analysis of the frequent retrievers ... was contaminated by reconstructive strategies" and this is what accounts for the significant response time (RT) size effect. Interestingly, the empirical case supporting the conventional wisdom is not clear-cut, in no small part, for the same reason.

Confounding responses from reconstructive strategies with those from a reproductive process has been a long-standing problem in developing and testing cognitive models of expert retrieval, including those representing the conventional wisdom. The network-retrieval model (Ashcraft, 1987), the distribution-ofassociations model (Siegler, 1988), and the networkinterference model (Campbell, 1987; Campbell \& Graham, 1985), for example, were all based on, or supported by, "retrieval" data that did not control for slow or fast reconstructive processes (Baroody, 1994). Consider, for example, Campbell's (1995) empirical test of the revised network-interference model/ simulation with 64 university students. The data appear to corroborate the model's central construct of "associative interference" (e.g., responding to $9+7$ with 15 or 17 -the sum of the related item $9+6$ and $9+8$, respectively). Unfortunately, Campbell (1995) reported no safeguards against the use of even conscious and relatively slow reconstructive strategies (i.e., self-reports or disqualifying time limits). Thus, his supporting evidence may have been largely or partially the by-product of misapplying a reconstructive strategy. For example, responding to $9+7$ with 15 or 17 may have been due to misapplying a counting strategy (e.g., losing track and counting on from 9 six or eight times instead of seven times) or a reasoning strategy (e.g., transforming 9 into 10 but not compensating for the addition of 1 to 9 by reducing 7 by 1 ). Responding to $4 \times 7$ with 21 or 35 may be due to losing track with a skip-count strategy (i.e., thinking “ $7,14,21$ " or “ $7,14,21,28,35$ ”).

In effect, existing cognitive models of mental addition are based on the common assumption that size effects are due to the architecture of the retrieval network. If size effects are entirely, or even largely, due to the sporadic use of reconstructive strategies as originally suggested by Groen and Parkman (1972) and reiterated by others (e.g., Baroody, 1994; Chen \& Campbell, 2017), then all current models are suspect and may need be revised. For example, if so, there is

\footnotetext{
${ }^{1}$ Thevenot, Barrouillet, Castel, and Uittenhove (2016) share this view. In their Footnote 1, Chen and Campbell (2017) imply that the term "reconstructive strategies" cannot apply to an automated procedure because Thevenot et al. (2016) used it to describe conscious addition procedures identified via selfreports. However, this conclusion appears to be based on the questionable premise that a self-reported strategy cannot be an automated one.
}

no need to invoke interference effects to account for size effects (Campbell, 1995).

Chen and Campbell (2017) concluded on p. 7: "The network interference model and computer simulation proposed by Campbell (1995; see also Campbell \& Oliphant, 1992; Whalen, 1997) predict precisely" Uittenhove et al.'s (2016) sum-related increase in RT for small addition problems. Given that the network interference model is probably not based on pure fact-recall data, the precise fit is a serious indictment of the model's validity. That is, the most plausible explanation for this precise fit is that both the network interference model and the size effect observed by Uittenhove et al. are based on data that confound reproductive and reconstructive strategy use.

\section{Why the case against compacted addition procedures (fast reconstructive strategies) is not convincing}

Chen and Campbell (2017) concluded that, consistent with the conventional wisdom, their team's recent evidence indicates that, with the exception of basic add-with-0 items, fast reconstructive processes do not play a role in expert mental addition of basic sums. They further implied that there is no evidence that reconstructive strategies (other than the rule for adding 0 ) become compacted.

\section{Campbell and colleagues' recent data}

Chen and Campbell (2017) present recent evidence based on their team's efforts to develop methods that differentiate between responses generated by reproductive and fast reconstructive processes. They concluded that their generalizationof-practice and retrieval-induced forgetting (RIF) data clearly favor direct recall (reproduction) of $n+1$ and $1+n$ and other non-zero combinations. Although Campbell and colleagues are to be commended for attempting to develop methods that differentiate between responses generated by reproductive and reconstructive processes, their results are not clear-cut.

No generalization of addition practice The premise of the generalization-of-practice paradigm is that practice with a subset of problems should speedup a fast reconstructive process, which should then increase its efficiency with a set of unpracticed items. For example, if a compacted counting procedure operates with simple addition with addends of 1 to 4 , then practicing a subset of such problems (e.g., $4+3$ ) should subsequently speed up performance with similar, unpracticed problems (e.g., 3 + 2). Chen and Campbell (2017) further argued that generalization in efficiency should be most evident with basic sums involving 1 , because such problems "require only a single counting element" (p. 10). They noted, though, that Campbell and Beech (2014) and a half dozen other studies have demonstrated a generalization-of-practice effect for basic $0+n$ sums but not for other basic sums, 
including $1+n$ items. Chen and Campbell concluded that such results indicate that generating basic add-with-0 sums entails a fast reconstructive process but that generating other sums, including those that entail adding with 1 , most likely involves an item-specific reproductive process. "Indeed," Chen and Campbell observed, "the substantial problem size effect for $n+1$ problems ..., a result predicted by the network interference model, is difficult to reconcile with a rule-based account" (p. 11).

However, the generalization-of-practice paradigm may not be sufficiently sensitive to distinguish between a reproductive process and all types of fast reconstructive processes. Transfer of practice will be most substantial IF all the items of the practice and transfer sets are solved by the same reconstructive process and have equivalent processing demands. Such is the case with the add-with- $0(n+0=n)$ rule, multiply-with- $0(n \times$ $0=0)$ rule, or multiply with $1(n \times 1=n)$ rule, because these algebraic rules specify which operand of an expression is the answer for every case. Moreover, the processing demands for applying, say, the add-with- 0 rule to $0+3$ and $0+7$ are the same - identify as the sum the non- 0 addend. The same cannot be said for other classes of basic combinations that may involve fast reconstructive processes.

Whereas reconstructive processes that involve algebraic rules provide the answer in the problem itself (Campbell, Dufour, \& Chen, 2015), other reconstructive or reasoningbased processes entail deducing the answer from known information - a process in which the task demands may vary across problems. Consider, for example, the add-with-1 rule: The sum of $n+1$ or $1+n$ is the number after $n$ in the counting sequence. Determining the sum of $1+3$ and $1+8$ each requires (a) understanding how the addition of 1 is related to the counting sequence (the add-with-1 rule) and (b) knowledge of a specific number-after relation. Whereas practice with, for instance, $1+4$ and $1+7$ may help to speed up the use of the add-with-1 rule, it would not facilitate the process of recalling the specific number-after relation for unpracticed items such as $1+3$ or $1+8$. Thus, a plausible alternative explanation for the lack of generalization to unpracticed $1+$ $n$ items is that practice does not speed-up the critical element of the add-with-1 reasoning process. Note that the distinction between algebraic rules and reasoning-based processes helps explain why response times with the add-with- 0 items are faster than those with add-with-1 combinations. Differences in processing demands may account for why there is a size effect for add-with-1 items. For instance, even adults may be more fluent with the number-after relations of a smaller number such as 3 than with a larger number such as 7 .

A similar argument applies to other fast reconstructive strategies for other categories of non-zero problems. For instance, practicing a near-doubles strategy with $2+3(2+[2+1]=[2+$ $2]+1=4+1=5$ ) would not appreciably transfer to using the same strategy with $4+5(4+[4+1]=[4+4]+1=8+1=9)$ for two reasons. Specifically, the two problems involve retrieving different doubles $(2+2$ and $4+4$, respectively) and different number-after $n$ relations (after 4 and after 8 , respectively). Note that if differential processing demands for the problems are not carefully equated for the practice set and the transfer set, item difficulty can be a confounding factor.

Balancing differential processing demands is a challenging task at best, because even among adults, there may be individual differences in which (fast) reconstructive strategy is used to solve a particular problem. Moreover, an individual adult's use of (fast) reconstructive strategies may vary across problems, even for a particular category of problems such as "small non-tie addition with sums to 10." For example, a participant might use counting-on or a skip-the-number-after- $n$ strategy (where $n=$ the larger addend) with $2+6$, a near-doubles strategy with $4+5$, and a create-a-doubles strategy with $4+6(4+[1+5]=[4+1]+5=5+5=10)$. Further weakening the impact of practice with non-zero problems, a participant's use of multiple reconstructive strategies dilutes practice with any one strategy. Even if the same reconstructive strategy is used across a class of problems, processing demands may not be equivalent. In brief, the generalization method may be sensitive to the effect of practice in the case of algebraic rules but not so in the cases of reasoning-based reconstructive processes. ${ }^{2}$

RIF of small non-tie additions The rationale for the RIF paradigm is that practice recalling one basic fact can subsequently interfere with the retrieval of a competing unpracticed fact. Chen and Campbell (2017) noted that a form of RIF provides "further converging evidence the [add-with-1] problems are solved by an item-specific process" (p. 10). Campbell et al. (2015) found, for example, that practicing both small addition non-ties such as $3+4$ and $1+n$ items such as $1+4$ slowed the retrieval of its related cross-operation counterpart such as $3 \times 4$ and $1 \times 4$, respectively. However, practicing $0+n=n$ and $0 \times n=0$ did not induce RIF with $0 \times n$ and $0+n$ items, respectively, because both types of items involve

\footnotetext{
${ }^{2}$ Results that raise questions about the reliability or sensitivity of the paradigm include: (1) Campbell and Beech (2014) concluded, "The procedure-based $0+$ $\mathrm{N}=\mathrm{N}$ problems presented clear evidence of generalization (i.e., practicing a subset of $0+\mathrm{N}$ problems lead to speed-up for a different subset of $0+\mathrm{N}$ problems), but there was no evidence of such generalization of practice for the nonzero problems. Although this was true of Block 1, it was not true of Block 2, in which practice on a first subset of items resulted in a 14-ms slowing of RT on a second subset of $0+\mathrm{N}$ items. (The RT for $1+\mathrm{N}$ and ties slowed 13 and $1 \mathrm{~ms}$, respectively, and the RT for small combinations speeded up $4 \mathrm{~ms}$. (2) The reliability and sensitivity of the paradigm is most likely compromised with basic sums in the teens, because even adults may use a greater variety of reconstructive strategies with such combinations than with other basic sums. Indeed, Campbell and Xue's (2001) participants reported reconstructive strategy for $25-50 \%$ of trials involving basic combinations with a sum greater than 10, and there was still no evidence of transfer. Chen and Campbell (2017) conceded that "generalization would be difficult to measure for these items ... because there is large RT variability for reconstructive strategies, and there are a variety of idiosyncratic procedures used for large additions" (p. 10).
} 
the use of rules rather than the retrieval of separately represented individual facts.

One concern with existing RIF evidence is the validity of the construct. The effects of RIF seem to be operating on a time frame beyond what one might expect for an automatic retrieval process. Fact recall is executed within a time frame of seconds, if not milliseconds. Is it plausible that a key component of such an automatic process would continue to operate many minutes later? For example, is it likely that a multiplication item, such as $2 \times 3$ practiced in the first $15 \mathrm{~min}$ of Campbell et al.'s (2015) experiment would create interference that would slow the recall of a related addition item, such as 2 +3 , presented in the last $15 \mathrm{~min}$ of the experiment (i.e., up to 30 min apart)? As currently assessed, it seems more plausible to attribute Campbell et al.'s (2015) RIF effects to a nonautomatic or conscious process. Replicating the RIF effects with related problems presented within a second apart would be an important validation of the method.

Another concern is that several predictions of the construct have not been empirically supported, and the post-hoc amendments are not entirely convincing.

1. Campbell, Chen, and Maslany (2013) found that Canadian and Chinese adults, as expected, exhibited RIF on addition ties and the former also did so with small non-ties. Unexpectedly, the Chinese participants did not exhibit RIF on small addition non-ties. Although Campbell et al. (2013) concluded that the more mathematically advanced Chinese participants might solve small addition problems by fast procedures instead of number-fact retrieval, Chen and Campbell (2017) offered a different explanation. As Chinese students often learn non-tie multiplication, but not addition, facts in a preferred operand order (smaller-operand first), "both problem encoding processes and the organization of the memory networks in Chinese adults might evolve quite differently for multiplication and addition" (p. 12). As RIF is competition driven, prior retrieval of a non-tie multiplication problem may not strongly activate its addition counterpart and not incite inhibition and RIF. Such an explanation is plausible if highly skilled Chinese adults did not learn the concepts of additive and multiplicative commutativity or these concepts had no impact on the internalization or mental representation of addition and multiplication combinations. Is likely, though, that they inflexibly memorized and retrieved all the non-tie multiplication facts as distinct facts?

2. Campbell et al. (2015) hypothesized that practicing $1+n$ (via fact retrieval) should not produce a RIF effect in the retrieval of rule-based $1 \times n$ problems and vice versa. They found that — contrary to their prediction - practicing $1+n$ items did produce a RIF effect with $1 \times n$ problems but that, consistent with their prediction, practicing $1 \times n$ items did not produce a RIF effect with $1+n$ problems. To explain the contrary finding, the authors concluded: "We propose that the practiced $1+\mathrm{N}$ items (e.g., $1+3=4,1+$ $5=6,1+6=7$ ) were individually strengthened during the practice phase and were readily reactivated by the $1 \times \mathrm{N}$ counterpart stimuli (e.g., $1 \times 3,1 \times 5,1 \times 6$ ) during the test phase. This cue-based interference blocked initiation of the item-general procedure required for the counterpart problems, producing item-specific RIF for $1 \times \mathrm{N}=\mathrm{N}$ problems" (p. 678). This involved post-hoc amendment to the model seems to rest on two questionable assumptions. One is that a single instance of retrieval will strengthen an addition combination substantially. Another is that a rule-based $1 \times n$ system readily reactivated the cue-based interference process of the separate fact-retrieval $1+n$ system?

Yet another concern is that Campbell and Metcalfe's (2007) finding that rule intrusions (e.g., $1+7=7$ ) are the most common error type for $1+n$ items seems inconsistent with Chen and Campbell's (2017) conclusion that a reproductive process is the most likely explanation for the retrieval of $n+1$ or $1+n$ sums. Why would rule intrusion be a factor if such items were not rule-based? Rule intrusion would seem more likely if both adding and multiplying with 1 were rule-based reconstructive processes.

\section{Other evidence that adults' efficient reconstructive strategies are not compacted}

Chen and Campbell (2017) allowed that evidence indicates relational knowledge plays an important role in adults' arithmetic strategy repertoire. Nevertheless, they concluded such evidence does not support the possibility of compacted procedures.

\section{Mediational strategies involving differences and quotients} Chen and Campbell (2017) note that adults can efficiently use addition- or multiplication-mediated strategies for determining differences and quotients, respectively. They conclude, however, "there is no evidence ... these are compacted procedures (i.e., automatic and unconscious); in fact, their use often appears in participants' self-reported strategies for subtraction and division (Campbell \& Agnew, 2009; Campbell \& Alberts 2009)" (p. 13). This conclusion is based on the questionable assumption that a normally automatic and nonconscious procedure cannot be consciously accessed. In fact, asking participants to self-report procedures may prompt them to consciously reflect on how they (non-consciously) solved a problem or to consciously start using a reconstructive strategy.

The question of the algebraic zero rule Chen and Campbell's (2017) argument that self-reporting a strategy disqualifies it as 
compacted procedure is also logically inconsistent with the conventional wisdom that algebraic $(n+0=n, n \times 0=0$, $n \times 1=n)$ are automatic and non-conscious. If algebraic rules can become compacted (but are still accessible to conscious thought), it stands to reason that other reconstructive strategies can, with practice, become so also.

Mediational strategies involving commutativity Contrary to some cognitive models, such as the distribution-ofassociations model (Siegler, 1987), Chen and Campbell (2017) allow that the additive (and multiplicative) commutativity may be incorporated into the retrieval system by representing, for example, both $3+5$ and $5+3$ non-redundantly, permitting each to be retrieved with equal efficiency. They concluded, "however, the specific perceptual or cognitive process by which North American adults rapidly map visually presented commuted pairs to a common memory representation remains unknown (Robert \& Campbell, 2008)" (p. 13). Given that the commutative relation affects all 81 of the basic non-zero combinations except for the nine ties, it seems premature to conclude that an item-specific reproductive process, rather than fast reconstructive processes, underlies the retrieval of the basic sums.

Mediational strategies not related to basic number combinations On p. 13, Chen and Campbell (2017) noted: "Given also that there appears to be little other evidence to support the idea that mediational strategies become proceduralized with practice (see Bajic \& Rickard, 2009; Kole \& Healy, 2013; Rickard, Lau \& Pashler, 2008), we conclude that the cumulative evidence for fast compacted procedures for simple addition ... is not convincing..." The cited references, though, have serious methodological limitations and provide unconvincing support for Chen and Campbell's (2017) conclusion. Both the Bajic and Rickard (2009) and Kole and Healy (2013) studies involved brief training and did not involve a delayed posttest. Thus, their results are not relevant to the issue of whether reconstructive strategies can, with practice over time, become represented in LTM as compacted procedures. Moreover, neither intervention involved structured and meaningful content and, thus, was not analogous to useful reconstructive strategies that embody arithmetic relations.

The Rickard et al. (2008) study at least involved a relatively complicated (1-digit $\times 2$-digit) arithmetic procedure and a posttest a week after the training. Although Experiment 1 entailed only a single session of 15 repetitions and Experiment 2 involved but two sessions of 30 repetitions, each experiment showed that both massed and distributed practice significantly decreased response time (i.e., the multiplication procedure became significantly more efficient). Rickard et al. unjustifiably characterized the shift in terms of a "transition from calculation to retrieval" from LTM. However, although
Experiment 2 participants self-reported their strategy (calculation, direct retrieval, or other) after each trial, Experiment 1 did not include a means of distinguishing between a reconstructive and reproductive strategy. More importantly, the results of both experiments might better be characterized as a transition from calculation to temporary (working) memory. As shown in Table 1, the gains (relatively fast RT and high percentage of direct recall) at the end of the training were largely lost on the first trial (Trial 31) of the delayed-test session and did not entirely recover by the eighth and last test trial, Trial 38. That is, the experiments did not appear to involve a transition from calculation to retrieval from LTM.

\section{Assumptions/issues that need to be addressed}

In addition to the need to investigate further operator priming, generalization effects, and attentional shifts (as suggested by Chen \& Campbell 2017), there are a number of other important and broader, but poorly understood, issues that require study (Baroody \& Purpura, 2017; Baroody \& Varma, 2006).

1. Does the retrieval system consist of an automatic reproductive process only or automatic reproductive AND reconstructive processes? Chen and Campbell (2017) argued that mental-addition expertise entails exclusively direct fact-recall (at least for non-zero basic sums) and imply that reconstructive strategies never achieve automaticity. As previously argued, it may not be safe to assume that reconstructive strategies cannot be automatized and be incorporated into the retrieval system. Indeed, the National Math Advisory Panel (NMAP, 2008) concluded that memory network of the retrieval system might be composed of both fact recall (a reproductive strategy) and efficient reasoning strategies (fast reconstructive strategies). For example, the fact that brain-damaged patients tended to recall or not recall commuted sums (rather than one or the other) is consistent with the proposition such sums are not represented discretely as individual facts but as a part-part-whole triplet (Sokol, McCloskey, Cohen, \& Aliminosa, 1991).

2. Among experts, might reconstructive and reproductive processes be coequals? Although there has been a "long-standing assumption that direct memory retrieval is ultimately the most efficient process of simple addition for non-zero problems" (Chen \& Campbell, 2017, p. 1; see also NMAP, 2008), the assumption is not clearly supported by empirical evidence - evidence that unquestionably and reliably distinguishes between reproductive and 
Table 1 Summary of Rickard et al.'s (2008) results for Experiment 2

Type of practice within a trial First trial of training session 1 Last trial of training session 2 Delayed test session

\begin{tabular}{|c|c|c|c|c|c|c|c|c|}
\hline & \multicolumn{2}{|l|}{ Trial 1} & \multicolumn{2}{|c|}{ Trial 30} & \multicolumn{2}{|c|}{ Trial 31} & \multicolumn{2}{|c|}{ Trial 38} \\
\hline & RT (s) & $\%$ of direct recall & RT (s) & $\%$ of direct recall & RT (s) & $\%$ of direct recall & RT (s) & $\%$ of direct recall \\
\hline Massed practice $^{\mathrm{a}}$ & $\sim 4.4$ & NR & $\sim 1.0$ & $\sim 82$ & $\sim 3.5$ & $\sim 10$ & $\sim 2.3$ & $\sim 50$ \\
\hline Distributed practice $^{\mathrm{b}}$ & $\sim 4.6$ & NR & $\sim 1.8$ & $\sim 58$ & $\sim 3.1$ & $\sim 25$ & $\sim 2.1$ & $\sim 55$ \\
\hline
\end{tabular}

$R T$ denotes response time, $N R$ indicates not reported

${ }^{a}$ For each trial, three items at time were practiced 15 times consecutively, then a second, third, and fourth set of three items were practiced in the same manner

${ }^{\mathrm{b}}$ For each trial, 12 intermixed items were practiced per trial

reconstructive processes. ${ }^{3}$ Moreover, "there is no a priori reason to assume that reconstructive strategies cannot be compacted and become as automatic and reliable as a reproductive strategy and perhaps with significantly less time and effort" (Baroody \& Varma, 2006). Indeed, several investigators have found that reconstructive processes were (significantly) more efficient than reproductive processes (Campbell \& Therriault, 2013; Fayol \& Thevenot, 2012; Metcalfe \& Campbell, 2011; Uittenhove et al., 2016). For example, the results of Campbell and Beech (2014) indicate that adults' responses to combinations involving 0 (which are generally perceived as rule-based) and those involving 1 (which may be rule-based) were faster than ties and, tie retrieval was not generally more accurate than that involving 0 or 1 .

3. Are representations of concepts or procedures and facts independent, interactive, or interdependent? Although it is commonly assumed non-reproductive and reproductive processes are represented in separate regions of the brain and operate independently (in a modular fashion), there are other possibilities that need to be systematically examined in both non-experts prior to compacting knowledge and experts with compacted knowledge. An interactive network would entail separate conceptual, procedural, and factual representations that cooperate or interact to determine basic sums (in a semi-modular fashion). For example, a commutativity schema would enable a child to recognize $3+4$ as equivalent to $4+3$ and access

\footnotetext{
${ }_{3}^{3}$ The consensus that a reproductive strategy (direct fact retrieval) is more efficient than reconstructive strategies is based on observations that young children miscalculate sums when they use counting strategies. Although children may be error-prone when first learning a counting strategy or using a cumbersome counting strategy, they may become relatively error-free after mastering an advanced counting strategy such as the relatively efficient MIN procedure or a reasoning strategy. Given associative confusions and other sources of memory failure/interference, it is not clear that a reproductive strategy is more efficient than a compacted reconstructive strategy. To my knowledge, there has not been a systematic examination of the issue - especially an examination that takes into account the cost/benefits ratio of achieving (near) perfect retrieval (including the amount of time and effort needed for such success).
}

the $4+3=7$ data node. An interdependent network would represent factual, conceptual, and procedural knowledge in an integrated fashion. For example, knowledge of commutativity would permit a single 3-4-7 representation, which would be triggered for $3+4$ or $4+3$.

4. Are different aspects of knowledge or processes accessed and applied serially (sequentially) or in parallel (simultaneously)? Consistent with Norman and Shallice's $(1980,1986)$ model, an interactive network might consist of a hierarchically organized network of action schemas that embody reconstructive processes, some of which then activitate a reproductive process. For example, Baroody and Ginsburg (1991) conjectured that an interactive network might involve serially activating a hierarchy of schemas in which the commutativity schema would have the highest priority: If the operation sign is plus, then addend order is irrelevant and focus on the key term (e.g., 0, 1, larger addend) regardless of its position. A small-then large addend problem would activate the lowest priority schema - a transformation schema that would equate $3+4$ with $4+3$. This process would then activate the recall of the factual knowledge $4+3=7$. In contrast, an integrated network of relational and factual knowledge would activate both types of knowledge simultaneously. Finally, an independent network might involve either serial reconstructive and reproductive processes (e.g., an initial attempt to recall a fact and, if unsuccessful, activation of a backup strategy) or parallel ones (e.g., simultaneously initiating a horse race between counting or reasoning strategies and direct fact recall). The evidence is unclear whether different aspects of knowledge or reconstructive and reproductive processes are implemented serially or in parallel. Compare, for instance, Jost, Hennighausen, and Rösler (2004) with Núñez-Peña, Cortiñas, and Escera (2006).

5. Is there only one course of development or expert state? As suggested by Chen and Campbell (2017), results can vary depending on the education background of participants. Delazer et al. (2005) found that different approaches to instruction (memorization by rote versus 
strategy-based instruction) produced differences in the location of the brain in which processing of the learned material took place. These results underscore the importance for researchers to consider the instructional background of participants before drawing general conclusions. For example, memory interference may vary depending on learning history - whether a class of combinations was memorized by rote or memorized meaningfully. Put differently, adults whose representation of sums entails integrated conceptual, procedural, factual knowledge may be less prone to associative confusion than those who memorized them as isolated facts. Delazer et al.'s results also indicate that the nature of expertise may vary among adults and depend on educational experiences. Put differently, separate models of retrieval may be needed for adults whose education focused on memorizing facts by rote and those who education focused on relational learning (Bisanz, 2003).

6. How does the retrieval network change as a result of learning? According to the conventional wisdom, the path to mental-addition expertise is marked by quantitative changes - increasing associative strength between addends and sum. However, might development also entail qualitative changes? For example, might learning the commutativity principle result in a mental reorganization of the basic sums (Butterworth, Marschesini, \& Girelli, 2003) - a shift from an independent to an interdependent representation? As conceptual, procedural, and factual knowledge becomes more integrated and compacted, might their mental representation become less modular?

\section{Conclusion}

Whether their model is ultimately corroborated, the proponents of the compacted counting view have made a number of important contributions. These include reviving the debate about the role of reconstructive processes in expert retrieval, questioning key assumptions of conventional cognitive models, and raising questions about commonly used research methods such as the limitations of self-reports. Chen and Campbell's (2017) careful and thoughtful critique of the research supporting a compacted counting model is a useful step in the enduring debate on how basic sums are mentally represented and processed. However, given the unclear empirical support for conventional cognitive models and the many unresolved issues about the nature and processing of the retrieval system, it seems more important than ever to pursue the debate and the research necessary to resolve it. For this reason, I look forward to hearing Uittenhove et al.'s (2016) response to Chen and Campbell's (2017) critique. I especially look forward to research that addresses the outstanding issues needed to construct a more accurate model of basic number combination learning and representation - a model that perhaps integrates reconstructive and reproductive processes in some fashion.

\section{References}

Ashcraft, M. H. (1982). The development of mental arithmetic: A chronometric approach. Developmental Review, 2, 213-236. https://doi. org/10.1016/0273-2297(82)90012-0

Ashcraft, M. H. (1987). Children's knowledge of simple arithmetic: A developmental model and simulation. In J. Bisanz, C. J. Brainer, \& R. Kail (Eds.), Formal methods in developmental psychology (pp. 302-338). New York: Springer-Verlag.

Bajic, D., \& Rickard, T. C. (2009). The temporal dynamics of strategy execution in cognitive skill learning. Journal of Experimental Psychology: Learning, Memory, and Cognition, 35, 113-121.

Baroody, A. J. (1983). The development of procedural knowledge: An alternative explanation for chronometric trends of mental arithmetic. Developmental Review, 3, 225-230. https://doi.org/10.1016/02732297(83)90031-X

Baroody, A. J. (1994). An evaluation of evidence supporting fact-retrieval models. Learning and Individual Differences, 6, 1-36. https://doi. org/10.1016/1041-6080(94)90013-2

Baroody, A. J., \& Ginsburg, H. P. (1991). A cognitive approach to assessing the mathematical difficulties of children labeled learning disabled. In H. L. Swanson (Ed.), Handbook on the assessment of learning disabilities: Theory, research, and practice (pp. 177-227). Boston: College-Hill Press.

Baroody, A. J., with Purpura, D. J. (2017). Early number and operations: Whole numbers. In J. Cai (Ed.), Compendium for research in mathematics education (pp. 308-354). Reston, VA: National Council of Teachers of Mathematics.

Baroody, A. J., \& Varma, S. (2006, November). The active construction view of basic number fact knowledge: New directions for cognitive neuroscience. Invited presentation at the Neural Basis of Mathematical Cognition Conference, Nashville, TN.

Barrouillet, P., \& Thevenot, C. (2013). On the problem-size effect in small additions: Can we really discard any counting-based account? Cognition, 128, 35-44. https://doi.org/10.1016/j.cognition.2013.02.018

Bisanz, J. (2003). Arithmetical development: Commentary on chapters 1 through 8 and reflections on directions. In A. J. Baroody \& A. Dowker (Eds.), The development of arithmetic concepts and skills: Constructing adaptive expertise (pp. 435-452). Mahwah, NJ: Erlbaum.

Butterworth, B., Marschesini, N., \& Girelli, L. (2003). Basic multiplication combinations: Passive storage or dynamic reorganization. In A. J. Baroody \& A. Dowker (Eds.), The development of arithmetic concepts and skills: Constructing adaptive expertise (pp. 189202). Mahwah, NJ: Erlbaum.

Campbell, J. I. D. (1987). Network interference and mental multiplication. Journal of Experimental Psychology: Learning, Memory and Cognition, 13, 109-123.

Campbell, J. I. D. (1995). Mechanisms of simple addition and multiplication: A modified network-interference theory and simulation. Mathematical Cognition, 1, 121-164.

Campbell, J. I. D., \& Agnew, H. (2009). Retrieval savings with nonidentical elements: The case of simple addition and subtraction. Psychonomics Bulletin and Review, 16, 934-944. https://doi.org/ 10.3758/PBR.16.5.938

Campbell, J. I. D., \& Alberts, N. M. (2009). Operation-specific effects of numerical surface form on arithmetic strategy. Journal of Experimental Psychology: Learning, Memory, and Cognition, 35, 999-1011. 
Campbell, J. I. D. \& Beech, L. C. (2014). No generalization of practice for non-zero simple addition. Journal of Experimental psychology: Learning, Memory, and Cognition, 40, 1766-1771. https://doi.org/ 10.1037/xlm0000003

Campbell, J. I. D., \& Graham, D. J. (1985). Mental multiplication skill: Structure, process, and acquisition. Canadian Journal of Psychology, 39, 338-366. https://doi.org/10.1037/h0080065

Campbell, J. I. D., \& Metcalfe, A. W. S. (2007). Numeral format and arithmetic rules. European Journal of Cognitive Psychology, 19, 335-355. https://doi.org/10.1080/09541440600717610

Campbell, J. I. D., \& Oliphant, M. (1992). Representation and retrieval of arithmetic facts: A network-interference theory and simulation. In J. I. D. Campbell (Ed.), The nature and origins of mathematical skills (pp. 331-364). Amsterdam: Elsevier.

Campbell, J. I. D., \& Therriault, N. H. (2013). Retrieval-induced forgetting of arithmetic facts but not rules. Journal of Cognitive Psychology. 25, 717-724. https://doi.org/10.1080/20445911.2013. 798328

Campbell, J. I. D., \& Xue, Q. (2001). Cognitive arithmetic across cultures. Journal of Experimental Psychology: General, 130, 299-315. https://doi.org/10.1037/0096-3445.130.2.299

Campbell, J. I. D., Chen, Y., \& Maslany, A. J. (2013). Retrieval-induced forgetting of arithmetic facts across cultures. Journal of Cognitive Psychology, 25, 759-773.

Campbell, J. I. D., Dufour, K. D., \& Chen Y. (2015). Retrieval-induced forgetting of multiplication facts and identity rule. Memory \& Cognition, 43, 672-680. https://doi.org/10.3758/s13421-014-0483-1

Chen, Y., \& Campbell, J. I. D. (2017). "Compacted" procedures for adults' simple addition: A review and critique of the evidence. Psychonomic Bulletin \& Review. https://doi.org/10.3758/s13423017-1328-2

Delazer, M., Ischebeck, A., Domahs, F., Zamarian, L., Koppelstaetter, F., Siedentopf, C. M., . . . Felber, S. (2005). Learning by strategies and learning by drill — evidence from an fMRI study. NeuroImage, 25 , 838-849. https://doi.org/10.1016/j.neuroimage.2004.12.009

Fayol, M., \& Thevenot, C. (2012). The use of procedural knowledge in simple addition and subtraction. Cognition, 123, 392-403. https:// doi.org/10.1016/j.cognition.2012.02.008

Groen, G. J., \& Parkman, J. M. (1972). A chronometric analysis of simple addition. Psychological Review, 79, 329-343.

Jost, K., Hennighausen, E., \& Rösler, F. (2004). Comparing arithmetic and semantic fact retrieval: Effects of problem size and sentence constraint on event related brain potentials. Psychophysiology, 41, 46-59. https://doi.org/10.1111/1469-8986.00119 411

Kole, J. A., \& Healy, A. F. (2013). Is retrieval mediated after repeated testing? Journal of Experimental Psychology: Learning, Memory, and Cognition, 39, 462-472. https://doi.org/10.1037/a0028880
Metcalfe, A. W. S., \& Campbell, J. I. D. (2011). Adults' strategies for simple addition and multiplication: Verbal self-reports and the operand recognition paradigm. Journal of Experimental Psychology: Learning, Memory, and Cognition, 37, 884-893. https://doi.org/ $10.1037 / \mathrm{a} 0022218$

National Mathematics Advisory Panel. (2008). Foundations for success: The final report of the National Mathematics Advisory Panel. Washington, DC: U.S. Department of Education.

Norman, D. A., \& Shallice, T. (1980). Attention to action: Willed and automatic control of behavior. (Technical Report No. 99). Center for Human Information Processing.

Norman, D. A., \& Shallice, T. (1986). Attention to action: Willed and automatic control of behavior. In R. J. Davidson, G. E. Schwartz, \& D. Shapiro (Eds.), Consciousness and self-regulation (pp. 1-18). New York: Plenum Press.

Núñez-Peña, M. I., Cortiñas, M., \& Escera, C. (2006). Problem size effect and processing strategies in mental arithmetic. NeuroReport, 17, 357-360. https://doi.org/10.1097/01.wnr.0000203622.24953.c2

Rickard, T. C., Lau, J. S., \& Pashler, H. (2008). Spacing and the transition from calculation to retrieval. Psychonomic Bulletin \& Review, 15, 656-661. https://doi.org/10.3758/PBR.15.3.656

Robert, N. D., \& Campbell, J. I. D. (2008). Simple addition and multiplication: No comparison. European Journal of Cognitive Psychology, 20, 123-138. https://doi.org/10.1080/09541440701275823

Siegler, R. S. (1987). Strategy choices in subtraction. In J. Slobada \& D. Rogers (Eds.), Cognitive process in mathematics (pp. 81-106). Oxford, England: Oxford University Press.

Siegler, R. S. (1988). Strategy choice procedures and the development of multiplication skill. Journal of Experimental Psychology: General, 117, 258-275. https://doi.org/10.1037/0096-3445.117.3.258

Sokol, S. M., McCloskey, M., Cohen, N. J., \& Aliminosa, D. (1991). Cognitive representations and processes in arithmetic: Inferences from the performance of brain-damaged subjects. Journal of Experimental Psychology: Learning, Memory, and Cognition, 17, $355-376$.

Thevenot, C., Barrouillet, P., Castel, C., \& Uittenhove, K. (2016). Tenyear-old children strategies in mental addition: A counting model account. Cognition, 146, 48-57. https://doi.org/10.1016/j.cognition. 2015.09.003

Uittenhove, K., Thevenot, C., \& Barrouillet, P. (2016). Fast automated counting procedures in addition problem solving: When are they used and why are they mistaken for retrieval? Cognition, 146, 289-303. https://doi.org/10.1016/j.cognition.2015.10.008

Whalen, J. (1997). The influence of semantic magnitude representations on arithmetic: Theory, data, and simulation. Proceedings of the Nineteenth Annual Conference of the Cognitive Science Society, USA, 19, 814-819. 